\title{
EFEITOS DA APLICAÇÃO DO ÁCIDO ASCÓRBICO EM MUDAS DE COUVE FOLHA (Brassica oleracea) CULTIVADAS EM DIFERENTES NÍVEIS SALINOS
}

\author{
Cinara Bernardo da Silva ${ }^{1}$, Cleice Fátima Gonçalves Alves², Julianna Catonio da Silva ${ }^{3}$, João Cardoso de \\ Albuquerque Neto ${ }^{4}$, Márcio Aurélio Lins dos Santos ${ }^{5}$, José Vieira Silva ${ }^{5}$
}

\begin{abstract}
'Engenheira Agrônoma, Mestre em Agricultura e Ambiente, Extensionista Emater/ Arapiraca-Al, Rua Governador Silvestre Péricles, № 1065, Jardim Tropical, Arapiraca/AL Brasil, CEP 57313-390

2Engenheira Agrônoma, Mestre em Agricultura e Ambiente, Extensionista Emater/ Arapiraca-Al, Avenida Getúlio Vargas, № 56, Centro, Penedo/AL, CEP 57200-000

${ }^{3}$ Engenheira Agrônoma, Mestre em Agricultura e Ambiente, Doutoranda em Produção Vegetal, Universidade Federal de Alagoas, Campus de Engenharias e Ciências Agrárias BR-104, Km 85, s/n. Rio Largo - AL CEP 57100-000

${ }^{4}$ Engenheiro Agrônomo, na Cooperativa de Trabalho dos Profissionais de Agronomia Ltda (UNICAMPO), Rua Paraíba, № 2490, Luís Eduardo Magalhães - BA, CEP: 47850-000

${ }^{5}$ Professor da Universidade Federal de Alagoas, Campus de Arapiraca, Avenida Manoel Severino Barbosa, S/N, Bom Sucesso, Arapiraca - AL, CEP 57309-005
\end{abstract}

RESUMO: Para minimizar as perdas de produtividade causadas pelo o uso de água de qualidade inferior, antioxidantes podem ser aplicados na forma exógena, sendo o Ácido Ascórbico (AsA) um dos mais importantes. Objetivou-se avaliar o efeito da aplicação de AsA via foliar para redução dos efeitos da salinidade na cultura da couve folha (Brassica oleracea L). 0 experimento foi conduzido na Universidade Federal de Alagoas (UFAL) - Campus de Arapiraca, em ambiente protegido. 0 delineamento experimental foi 0 inteiramente casualizado, esquema fatorial $4 \times 3$, com 5 repetições. Utilizou-se níveis de salinidade (S1: 0,12; S2: 1,62; S3: 2,62 e S4: 4,12 dS. $\mathrm{m}^{-1}$ ) e doses de AsA (A1:0; A2: 5 e A3: $20 \mathrm{mM}$ ). Analisou-se: altura de planta (AP), número de folhas (NF) e comprimento da raiz (CR). $O$ nível de tolerância da couve folha à salinidade foi de 1,62 dS. $\mathrm{m}^{-1}$, porém a AP e o CR não foram afetados até os níveis de 2,2 e 4,2 dS. $\mathrm{m}^{-1}$, respectivamente. $O$ uso exógeno de AsA mostrou-se pouco promissor ou recomendável para reverter ou minorar os efeitos da salinidade sobre o crescimento de couve folha.

PALAVRAS CHAVE: Recursos hídricos; antioxidantes; salinidade.

\section{EFFECTS OF THE APPLICATION OF ASCORBIC ACID ON LEAF (Brassica oleracea) SEEDLINGS GROWN IN DIFFERENT SALIN LEVELS}

\begin{abstract}
To minimize the productivity losses caused by saline water use, exogenous antioxidants such as Ascorbic Acid (AsA) can be applied. The objective was to evaluate the leaf application of AsA to reduce the salinity effects on the leaf cabbage (Brassica oleracea $L$ ) culture. The experiment was conducted at the Federal University of Alagoas (UFAL) - Arapiraca Campus, in a protected environment. The experimental design was a completely randomized, $4 \times 3$ factorial scheme, with 5 replications. Salinity levels (S1: 0.12; S2: 1.62; S3: 2.62 and S4: 4.12 $\mathrm{dS} . \mathrm{m}^{-1}$ ) and AsA doses (A1: 0; $\mathrm{A} 2: 5$ and $\mathrm{A} 3: 20 \mathrm{mM}$ ) were used. It was analyzed: plant height (PH), leaves number $(\mathrm{LN})$ and root length (RL). The tolerance level of leaf cabbage to salinity was $1.62 \mathrm{dS} . \mathrm{m}^{-1}$, but PH and RL were not affected until the levels of 2.2 and $4.2 \mathrm{dS} . \mathrm{m}^{-1}$, respectively. The AsA exogenous use was not very promising or recommended to reverse or reduce the salinity effects on the growth of leaf cabbage.
\end{abstract}

KEY WORDS: Water resources; antioxidants; salinity.

\section{INTRODUÇÃO}

A couve folha (B. oleracea L.) pertence à família Brassicaceae é rica em nutrientes como ferro, cálcio, vitamina $\mathrm{A}$ e ácido ascórbico, sendo também uma ótima fonte de carotenoides apresentando, entre as hortaliças, a maior concentração de luteína e de beta caroteno (Filgueira, 2013).

A produção da couve em Alagoas é bem aceita pelos produtores da região, mas vale salientar que ocorre uma irregularidade na distribuição das chuvas 
o que ocasiona em uma má distribuição de água na região. A água de qualidade encontra-se cada vez mais escassa forçando os produtores na utilização de água de qualidade inferior. Entretanto, a utilização adequada desse tipo de água é fundamental para reduzir os danos que podem ser causados às plantas e ao solo.

As plantas, quando submetidas ao estresse salino, podem apresentar diferentes respostas, sendo esse fenômeno extremamente complexo, envolvendo alterações morfológicas e de crescimento, além de processos fisiológicos e bioquímicos. As alterações no metabolismo induzidas pela salinidade são resultado de várias respostas fisiológicas da planta, dentre as quais se destacam as modificações no crescimento, comportamento estomático e capacidade fotossintética (Garcia et al., 2010; Paiva, 2017).

Existem estudos afim de reduzir os efeitos dos sais nas plantas, o Ácido Ascórbico, no entanto, é um dos antioxidantes mais importantes na proteção de plantas contra 0 estresse oxidativo gerado por estresse abiótico (Castro, 2014). A aplicação do Ácido Ascórbico como antioxidante (AsA) endógeno elevado em plantas é necessário para neutralizar o estresse oxidativo, além de outros processos do metabolismo vegetal. O Ácido Ascórbico (AsA) desempenha papel chave na tolerância ao sal de muitas plantas halófitas e é prontamente absorvido após a aplicação exógena movendo-se dentro da planta, sendo a aplicação foliar de AsA capaz de melhorar a tolerância das plantas aos sais de alguma maneira (Athar; Khan; Ashraf; 2008; Hameed et al., 2015).

Objetivou-se avaliar o efeito da aplicação de Ácido Ascórbico via foliar na redução dos efeitos da salinidade na cultura da couve folha (Brassica oleracea L).

\section{MATERIAL E MÉTODOS}

O experimento foi conduzido na Universidade Federal de Alagoas (UFAL) - Campus de Arapiraca, em ambiente protegido, com as coordenadas geodésicas $9^{\circ}$ $45^{\prime} 09^{\prime \prime}$ de latitude Sul e $36^{\circ} 39^{\prime} 40^{\prime \prime}$ de longitude Oeste e altitude de $325 \mathrm{~m}$, no período de maio a junho de 2018 .

Adotou-se 0 delineamento inteiramente casualizado (DIC), em esquema fatorial $4 \times 3 \mathrm{com} 5$ repetições. Os tratamentos consistiram de níveis de salinidade, (S1: 0,12; S2: 1,62; S3: 2,62 e S4: 4,12 dS $\mathrm{m}^{-1}$ de condutividade elétrica da água de irrigação) e três doses do ácido ascórbico (A1:0; $A 2$ : 5 e A3: 20 $\mathrm{mM}$, onde $\mathrm{A} 2:$ : 0,22 g e A3: 0,73 g do ácido).

Preparou-se o substrato para as plantas, usando solo argiloso e casca de arroz em uma proporção de $1 / 1$, realizou-se a mistura utilizando a água contendo $\left(0,12 \mathrm{dSm}^{-1}\right)$. Os tratamentos foram dispostos em bandejas de plásticos, sendo a parcela representada por 10 células e avaliadas as três plantas centrais. As bandejas foram irrigadas utilizando os níveis salinos, aplicando em cada célula, $20 \mathrm{~mL}$ da água diariamente. Já a aplicação do ácido realizou-se a cada $48 \mathrm{~h}$, via foliar utilizando um borrifador. A avaliação ocorreu 30 dias após a aplicação dos tratamentos (DAT).

As variáveis analisadas foram: altura de planta (AP), número de folhas (NF) e comprimento da raiz (CR). Os dados foram tabulados e submetidos à análise de variância ANAVA, utilizando o software estatístico SISVAR (Ferreira, 2011).

\section{RESULTADOS E DISCUSSÃO}

Observou-se que houve efeito significativo nos níveis de salinidade e aplicações de ácido ascórbico (AsA) afetando significativamente as variáveis: altura da planta (AP), número de folhas (NF) e comprimento da raiz (CR) (Tabela 1).

Tabela 1. Resumo da análise de variância para altura da planta (AP), número de folhas (NF) e área foliar (CR) na cultura da Couve.

\begin{tabular}{lcccc}
\hline \multirow{2}{*}{ Fator de variação } & \multirow{2}{*}{ GL } & \multicolumn{3}{c}{ VALORES DE QUADRADOS MÉDIOS } \\
\cline { 3 - 5 } & & AP & NF & CR \\
\hline Salinidade & 3 & $1,13^{\text {ns }}$ & $0,34^{\text {ns }}$ & $78,96^{* *}$ \\
Ácido Ascórbico & 2 & $0,14^{\text {ns }}$ & $0,57^{*}$ & $29,78^{* *}$ \\
SxA & 6 & $5,63^{* *}$ & $0,20^{\text {ns }}$ & $65,22^{* *}$ \\
Erro & 48 & 1,37 & 0,14 & 5,22 \\
CV $(\%)$ & & 11,31 & 11,99 & 15,53 \\
\hline
\end{tabular}

${ }^{*} \mathrm{e}^{* *}$ significativo a 5 e $1 \%$ de probabilidade, respectivamente; NS não significativo 
É possível observar que a planta não sofreu decréscimo na sua altura até $2,2 \mathrm{dS} \cdot \mathrm{m}^{-1}$ (Figura 1A). Contudo, o posterior aumento dos níveis salinos reduziu o crescimento da couve em até $39 \%$. O ácido ascórbico promoveu efeito quadrático apenas nas salinidades de 0,12 e 1,62 dS. $\mathrm{m}^{-1}$. Contudo, na salinidade de menor nível, a aplicação de $5 \mathrm{mM}$ de ácido ascórbico aumentou a altura da planta, enquanto no nível salino de $1,62 \mathrm{dS} \cdot \mathrm{m}^{-1}$ reduziu 0 crescimento da planta. Além disso, a aplicação de $20 \mathrm{mM}$ de ácido ascórbico ocasionou uma redução mínima de altura de $11 \%$ entre os níveis 0,12 e 1,62 dS. $\mathrm{m}^{-1}$ (Figura 1B). Embora os níveis salinos $0,12 \mathrm{e}$ $1,62 \mathrm{dS}$. $\mathrm{m}^{-1}$ não representem intolerância na planta, 0 ácido ascórbico pode ter apresentado efeito negativo quando aplicado na concentração de 20 mM.

Figura 1. Altura da planta em função da salinidade da água de irrigação (A) e Altura planta em função da salinidade da água de irrigação e concentração de ácido ascórbico (B).

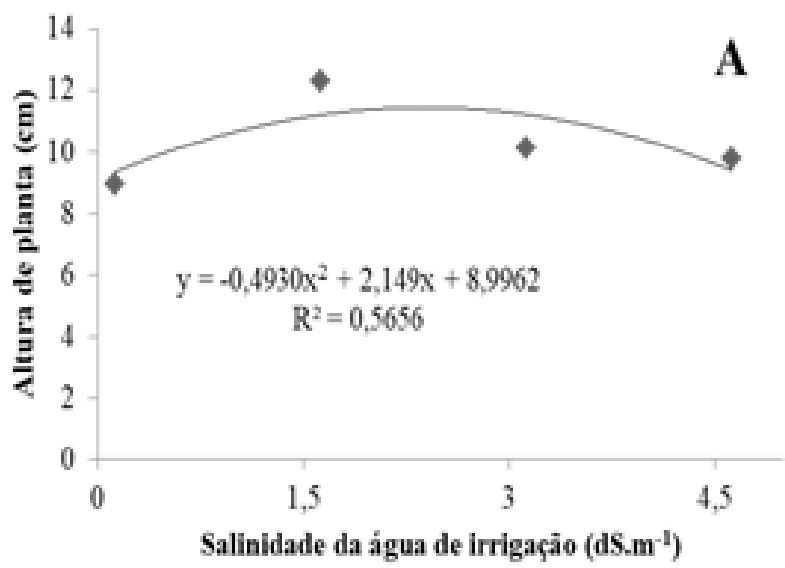

Segundo Dias e Blanco (2010), os efeitos da salinização sobre as plantas podem ser causados pela dificuldade de absorção de água, toxicidade de íons específicos e pela interferência dos sais nos processos fisiológicos, reduzindo o crescimento e desenvolvimento das plantas.

Para a variável número de folhas, com o aumento do ácido ocorreu um crescimento e posteriormente um decrescimento (Figura 2). 0 crescimento máximo do número de folhas ocorreu com a aplicação de ácido ascórbico de 14,47 (mM), obtendo uma média de 3 folhas.

Figura 2. Resposta do número de folhas (unidades), sob diferentes doses de ácido ascórbico.

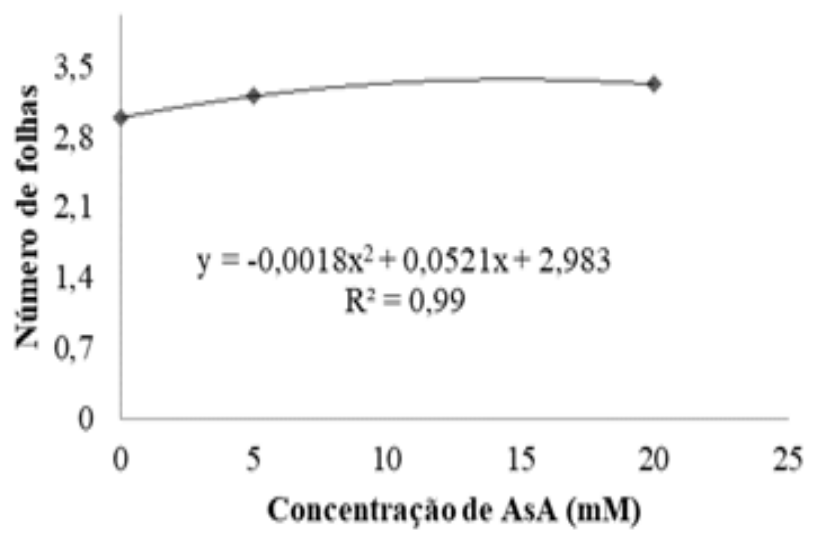

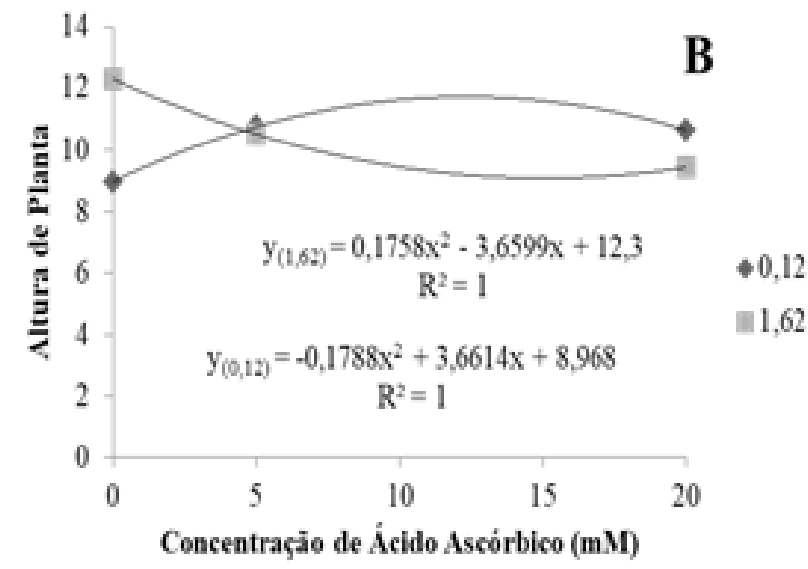

Maia (2017) observou que com exceção do número de folhas, todas as variáveis da alface crespa sofreram influência da interação dos fatores salinidade e Ácido ascórbico. Tal fato pode ocorrer, uma vez que o número de folhas depende da formação e desenvolvimento de primórdios foliares. Assim, a formação e o desenvolvimento dos primórdios foliares foram mais sensiveis ao estresse salino do que a expansão das folhas tanto através da redução na pressão de turgescência como na extensibilidade da parede celular (Prisco, 1980).

No desdobramento da salinidade dentro de cada ácido, o comprimento da raiz, cresceu de acordo com 0 aumento do nível de salinidade, até $1,30 \mathrm{dS} \mathrm{m}^{-1}$ (Figura $3 \mathrm{~A}$ ). Já no desdobramento do ácido dentro de cada salinidade, com a aplicação do ácido Ascórbico ocorreu um aumento do comprimento da raiz de acordo com o aumento da aplicação do ácido (Figura 3 B).

Segundo Athar et al., (2008) a aplicação do Ácido Ascórbico no enraizamento neutraliza os efeitos adversos do estresse salino sobre o crescimento, melhorando a capacidade fotossintética e mantendo a homeostase dos íons. 
Figura 3. Desdobramento sob diferentes níveis de salinidade da água de irrigação (dS.m-1) dentro de cada dose de ácido, na variável do comprimento de raiz (CR) (A) e desdobramento da concentração de $A s A(m M)$ dentro de cada nível de salinidade na variável do comprimento de raiz $(B)$.

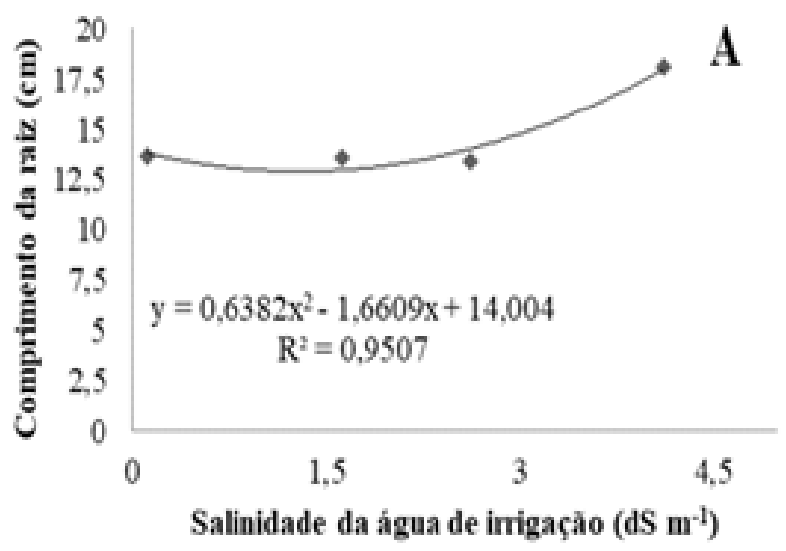

Observou-se que a utilização de ácido ascórbico para minimizar o efeito da salinidade na produção de mudas de couve não é recomendado, pois ao aumentar a salinidade não houve resultado positivo na utilização do ácido ascórbico. Sendo a tolerância da couve a salinidade de $1,62 \mathrm{dS} \cdot \mathrm{m}^{-1}$.

\section{REFERÊNCIAS BIBLIOGRÁFICAS}

Athar, H.; Khan, A.; Ashraf, M. Exogenously applied ascorbic acid alleviates salt-induced oxidative stress in wheat. Environmental and Experimental Botany, 2008, $63,1,224-231$.

Castro, J. L. S. A toxidade do ácido ascórbico em plantas de arroz silenciadas nas APXs cloroplásticas induz estresse oxidativo não dependente da fotossíntese. Fortaleza, 2014. 80p. Tese (doutorado em bioquímica) - Universidade Federal do Ceará, UFC.

Dias, N. S.; Blanco, F. F. Manejo da salinidade na agricultura: estudos básicos e avançados. Instituto Nacional de Ciência e Tecnologia em Salinidade. 2010, Fortaleza, 11p.

Ferreira, D.F. Sisvar: a computer statistical analysis system. Ciência e Agrotecnologia, 2011, 35, 1039-1042.

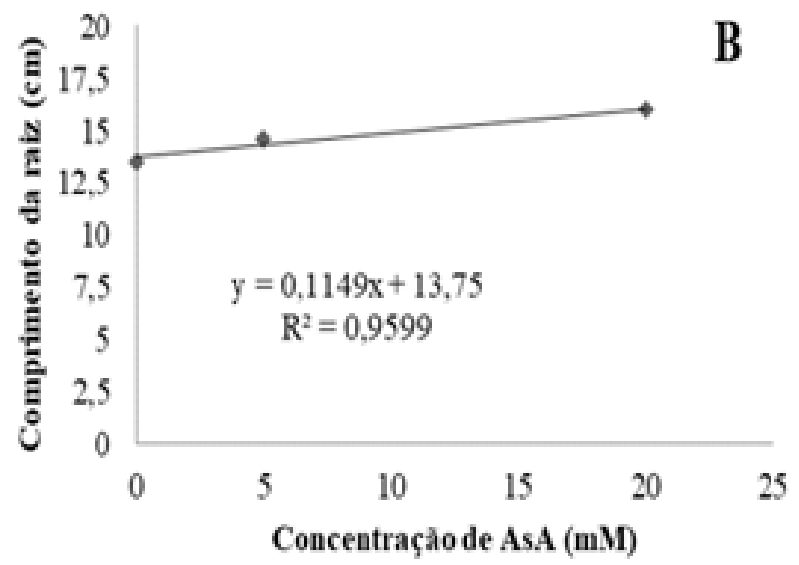

Filgueira, F. A. R. Novo manual de olericultura: agrotecnologia moderna na produção e comercialização de hortaliças. 2013, Viçosa: Editora UVF, p. 421.

Garcia, G. O; Nazário, A. A.; Moraes, W. B.; Gonçalves, I. Z.; Madalão, J. C. Respostas de genótipos de feijoeiro á salinidade. Engenharia na Agricultura, 2010, 18, 4, 330-338.

Hameed, A.; Gulzar, S.; Aziz, I.; Hussain, T.; Gul, B.; Khan, M. A. Effects of salinity and ascorbic acid on growth, water status and antioxidant system in a perennial halophyte. AoB Plants, 2015, 7, 4, 1-11.

Maia, P. DE M. E. Cultivo de hortaliças em substrato de fibra de coco sob estresse salino e aplicação foliar de ácido ascórbico. Minas Gerais, 2017. Tese (doutorado Recursos Hídricos em Sistemas Agrícolas) - Universidade Federal de Lavras, UFLA.

Paiva, F. I. G. Manejo da fertirrigação potássica e cálcica na cultura do tomateiro cultivadas em ambiente protegido e submetidas ao estresse salino. Mossoró, 2017. Dissertação (Mestrado em Manejo de Solo e Água) - Universidade Federal Rural do Semiárido, UFERSA.

Prisco, J. T. Alguns aspectos da fisiologia do "stress" Salino. Revista Brasileira de Botânica, 1980, 3, 85-94. 